\title{
On mixed boundary value problem of impulsive semilinear evolution equations of fractional order
}

\author{
Lihong Zhang ${ }^{1}$, Guotao Wang ${ }^{1 *}$ and Guangxing Song ${ }^{2}$
}

\footnotetext{
* Correspondence: wgt2512@163. com

${ }^{1}$ School of Mathematics and Computer Science, Shanxi Normal University, Linfen, Shanxi 041004, P. R. China

Full list of author information is available at the end of the article
}

\begin{abstract}
This article studies the existence and uniqueness of solutions for impulsive semilinear evolution equations of fractional order $\alpha \in(1,2]$ with mixed boundary conditions. Some standard fixed point theorems are applied to prove the main results. An illustrative example is also presented.

Mathematics Subject Classification: 26A33; 34K30; 34K45.
\end{abstract}

Keywords: evolution equations of fractional order, impulse, mixed boundary conditions, fixed point theorem.

\section{Introduction and preliminaries}

Fractional differential equations arise in many engineering and scientific disciplines as the mathematical modeling of systems and processes in the fields of physics, chemistry, aerodynamics, control theory, signal, and image processing, biophysics, electrodynamics of complex medium, polymer rheology, fitting of experimental data, etc. [1-6]. For example, one could mention the problem of anomalous diffusion [7-9], the nonlinear oscillation of earthquake can be modeled with fractional derivative [10], and fluiddynamic traffic model with fractional derivatives [11] can eliminate the deficiency arising from the assumption to continuum traffic flow and many other [12,13] recent developments in the description of anomalous transport by fractional dynamics. For some recent development on nonlinear fractional differential equations, see [14-24] and the references therein.

Impulsive differential equations, which provide a natural description of observed evolution processes, are regarded as important mathematical tools for the better understanding of several real world problems in applied sciences. The theory of impulsive differential equations of integer order has found its extensive applications in realistic mathematical modelling of a wide variety of practical situations and has emerged as an important area of investigation. The impulsive differential equations of fractional order have also attracted a considerable attention and a variety of results can be found in the articles [25-36]. 
Motivated by Agarwal and Ahmad's work [33], in this article, we study a mixed boundary value problem for impulsive evolution equations of fractional order given by

$$
\left\{\begin{array}{l}
{ }^{c} D^{\alpha} u(t)=A(t) u(t)+f(t, u(t)), \quad 1<\alpha \leq 2, \quad t \in J^{\prime}, \\
\Delta u\left(t_{k}\right)=I_{k}\left(u\left(t_{k}\right)\right), \quad \Delta u^{\prime}\left(t_{k}\right)=I_{k}^{*}\left(u\left(t_{k}\right)\right), \quad k=1,2, \ldots, p, \\
T u^{\prime}(0)=-a u(0)-b u(T), \quad T u^{\prime}(T)=c u(0)+d u(T), \quad a, b, c, d \in \mathbb{R},
\end{array}\right.
$$

where ${ }^{C} D^{\alpha}$ is the Caputo fractional derivative, $A(t)$ is a bounded linear operator on $J$ (the function $t \rightarrow A(t)$ is continuous in the uniform operator topology),

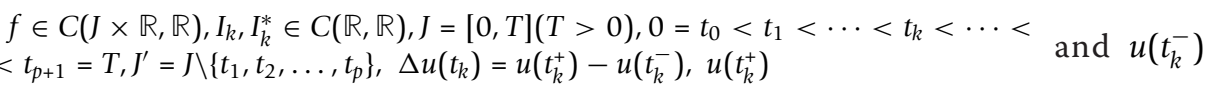
denote the right and the left limits of $u(t)$ at $t=t_{k}(k=1,2, \ldots, p)$, respectively and $\Delta u^{\prime}\left(t_{k}\right)$ have a similar meaning for $u^{\prime}(t)$.

It is worthwhile pointing out that the boundary conditions in (1.1) interpolate between Neumann $(a=b=c=d=0)$ and Dirichlet $(a, d \rightarrow \infty$ with finite values of $b$ and $c$ ) boundary conditions. Note that Zaremba boundary conditions $\left(u(0)=0, u^{\prime}(T)=\right.$ 0 ) can be considered as mixed boundary conditions with $a \rightarrow \infty, c=d=0$. For more details on Zaremba boundary conditions, see ( [37-39]).

Let $J_{0}=\left[0, t_{1}\right], J_{1}=\left(t_{1}, t_{2}\right], \ldots, J_{p-1}=\left(t_{p-1}, t_{p}\right], J_{p}=\left(t_{p}, T\right]$, and we introduce the spaces: $P C(J, \mathbb{R})=\left\{u: J \rightarrow \mathbb{R} \mid u \in C\left(J_{k}\right), k=0,1, \ldots, p\right.$, and $u\left(t_{k}^{+}\right)$exist, $\left.k=1,2, \ldots, p,\right\}$ with the norm $\|u\|=\sup _{t \in J}|u(t)|$, and $P C^{1}(J, \mathbb{R})=\left\{u: J \rightarrow \mathbb{R} \mid u \in C^{1}\left(J_{k}\right), k=0,1, \ldots, p\right.$, and $u\left(t_{k}^{+}\right), u^{\prime}\left(t_{k}^{+}\right)$exist, $\left.k=1,2, \ldots, p,\right\}$ with the norm $\|u\|_{P C^{1}}=\max \left\{\|u\|,\left\|u^{\prime}\right\|\right\}$. Obviously, $P C(J, \mathbb{R})$ and $P C^{1}(J, \mathbb{R})$ are Banach spaces.

Definition 1.1 A function $u \in P C^{1}(J, \mathbb{R})$ with its Caputo derivative of order $\alpha$ existing on $J$ is a solution of (1.1) if it satisfies (1.1).

For convenience, we give some notations:

$$
\begin{aligned}
& \lambda_{1}(t)=\frac{(b+d) T+(a d-b c) t}{\Lambda T}, \quad \lambda_{2}(t)=\frac{(b+1) T+(a+b) t}{\Lambda}, \\
& \lambda_{3}(t)=\frac{(1-d)(T+a t)+b(c+1) t}{\Lambda}, \quad \lambda_{4}=\frac{|a d|+|b c|}{T|\Lambda|}, \quad \lambda_{5}=\frac{|a+b|}{|\Lambda|}, \quad A_{1}=\max _{t \in J}|A(t)|,
\end{aligned}
$$

where $\Lambda=(b+1)(c+d)-(a+b)(d-1) \neq 0$.

Lemma 1.1 [26]For a given $y \in C[0, T]$, a function $u$ is a solution of the impulsive mixed boundary value problem

$$
\left\{\begin{array}{l}
{ }^{C} D^{\alpha} u(t)=y(t), \quad 1<\alpha \leq 2, \quad t \in J^{\prime}, \\
\Delta u\left(t_{k}\right)=I_{k}\left(u\left(t_{k}\right)\right), \quad \Delta u^{\prime}\left(t_{k}\right)=I_{k}^{*}\left(u\left(t_{k}\right)\right), \quad k=1,2, \ldots, p, \\
T u^{\prime}(0)=-a u(0)-b u(T), \quad T u^{\prime}(T)=c u(0)+d u(T), \quad a, b, c, d \in \mathbb{R},
\end{array}\right.
$$


if and only if $u$ is a solution of the impulsive fractional integral equation

$$
u(t)=\left\{\begin{array}{l}
\int_{0}^{t} \frac{(t-s)^{\alpha-1}}{\Gamma(\alpha)} \gamma(s) d s+\lambda_{1}(t) \int_{t_{p}}^{T} \frac{(T-s)^{\alpha-1}}{\Gamma(\alpha)} \gamma(s) d s \\
-\lambda_{2}(t) \int_{t_{p}}^{T} \frac{(T-s)^{\alpha-2}}{\Gamma(\alpha-1)} y(s) d s+A, \quad t \in J_{0} ; \\
\int_{t_{k}}^{t} \frac{(t-s) \alpha-1}{\Gamma(\alpha)} \gamma(s) d s+\lambda_{1}(t) \int_{t_{p}}^{T} \frac{(T-s)^{\alpha-1}}{\Gamma(\alpha)} \gamma(s) d s \\
-\lambda_{2}(t) \int_{t_{p}}^{T} \frac{(T-s)^{\alpha-2}}{\Gamma(\alpha-1)} \gamma(s) d s+\sum_{i=1}^{k}\left[\int_{t_{i-1}}^{t_{i}} \frac{\left(t_{i}-s\right)^{\alpha-1}}{\Gamma(\alpha)} \gamma(s) d s+I_{i}\left(u\left(t_{i}\right)\right)\right] \\
+\sum_{i=1}^{k-1}\left(t_{k}-t_{i}\right)\left[\int_{t_{i-1}}^{t_{i}} \frac{\left(t_{i}-s\right)^{\alpha-2}}{\Gamma(\alpha-1)} \gamma(s) d s+I_{i}^{*}\left(u\left(t_{i}\right)\right)\right] \\
+\sum_{i=1}^{k}\left(t-t_{k}\right)\left[\int_{t_{i-1}}^{t_{i}} \frac{\left(t_{i}-s\right)^{\alpha-2}}{\Gamma(\alpha-1)} \gamma(s) d s+I_{i}^{*}\left(u\left(t_{i}\right)\right)\right]+A, \quad t \in J_{k,} \quad k=1,2, \ldots, p,
\end{array}\right.
$$

where

$$
\begin{aligned}
A= & \lambda_{1}(t) \sum_{i=1}^{p}\left[\int_{t_{i-1}}^{t_{i}} \frac{\left(t_{i}-s\right)^{\alpha-1}}{\Gamma(\alpha)} \gamma(s) d s+I_{i}\left(u\left(t_{i}\right)\right)\right] \\
& +\lambda_{1}(t) \sum_{i=1}^{p-1}\left(t_{p}-t_{i}\right)\left[\int_{t_{i-1}}^{t_{i}} \frac{\left(t_{i}-s\right)^{\alpha-2}}{\Gamma(\alpha-1)} \gamma(s) d s+I_{i}^{*}\left(u\left(t_{i}\right)\right)\right] \\
& -\sum_{i=1}^{p}\left[\lambda_{3}(t)+\lambda_{1}(t) t_{p}\right]\left[\int_{t_{i-1}}^{t_{i}} \frac{\left(t_{i}-s\right)^{\alpha-2}}{\Gamma(\alpha-1)} \gamma(s) d s+I_{i}^{*}\left(u\left(t_{i}\right)\right)\right] .
\end{aligned}
$$

\section{Uniqueness and existence results}

Define an operator $T: P C(J, \mathbb{R}) \rightarrow P C(J, \mathbb{R})$ as

$$
\begin{aligned}
T u(t)= & \int_{t_{k}}^{t} \frac{(t-s)^{\alpha-1}}{\Gamma(\alpha)}(f(s, u(s))+A(s) u(s)) d s \\
& +\lambda_{1}(t) \int_{t_{p}}^{T} \frac{(T-s)^{\alpha-1}}{\Gamma(\alpha)}(f(s, u(s))+A(s) u(s)) d s \\
& -\lambda_{2}(t) \int_{t_{p}}^{T} \frac{(T-s)^{\alpha-2}}{\Gamma(\alpha-1)}(f(s, u(s))+A(s) u(s)) d s \\
& +\sum_{i=1}^{k}\left[\int_{t_{i-1}}^{t_{i}} \frac{\left(t_{i}-s\right)^{\alpha-1}}{\Gamma(\alpha)}(f(s, u(s))+A(s) u(s)) d s+I_{i}\left(u\left(t_{i}\right)\right)\right] \\
& +\sum_{i=1}^{k-1}\left(t_{k}-t_{i}\right)\left[\int_{t_{i-1}}^{t_{i}} \frac{\left(t_{i}-s\right)^{\alpha-2}}{\Gamma(\alpha-1)}(f(s, u(s))+A(s) u(s)) d s+I_{i}^{*}\left(u\left(t_{i}\right)\right)\right] \\
& +\sum_{i=1}^{k}\left(t-t_{k}\right)\left[\int_{t_{i-1}}^{t_{i}} \frac{\left(t_{i}-s\right)^{\alpha-2}}{\Gamma(\alpha-1)}(f(s, u(s))+A(s) u(s)) d s+I_{i}^{*}\left(u\left(t_{i}\right)\right)\right] \\
& +\lambda_{1}(t) \sum_{i=1}^{p}\left[\int_{t_{i-1}}^{t_{i}} \frac{\left(t_{i}-s\right)^{\alpha-1}}{\Gamma(\alpha)}(f(s, u(s))+A(s) u(s)) d s+I_{i}\left(u\left(t_{i}\right)\right)\right] \\
& +\lambda_{1}(t) \sum_{i=1}^{p-1}\left(t_{p}-t_{i}\right)\left[\int_{t_{i-1}}^{t_{i}} \frac{\left(t_{i}-s\right)^{\alpha-2}}{\Gamma(\alpha-1)}(f(s, u(s))+A(s) u(s)) d s+I_{i}^{*}\left(u\left(t_{i}\right)\right)\right] \\
& -\sum_{i=1}^{p}\left[\lambda_{3}(t)+\lambda_{1}(t) t_{p}\right]\left[\int_{t_{i-1}}^{t_{i}} \frac{\left(t_{i}-s\right)^{\alpha-2}}{\Gamma(\alpha-1)}(f(s, u(s))+A(s) u(s)) d s+I_{i}^{*}\left(u\left(t_{i}\right)\right)\right] .
\end{aligned}
$$


Lemma 2.1 The operator $T: P C(J, \mathbb{R}) \rightarrow P C(J, \mathbb{R})$ is completely continuous.

Proof. Observe that $T$ is continuous in view of the continuity of $f, I_{k}$ and $I_{k}^{*}$. Let $\Omega \subset$ $P C(J, \mathbb{R})$ be bounded, where $\Omega=\{u \in P C(J, \mathbb{R}):\|u\| \leq r\}$. Then, there exist positive constants $L_{i}>0(i=1,2,3)$ such that $|f(t, u(t))| \leq L_{1},\left|I_{k}(u)\right| \leq L_{2}$ and $\left|I_{k}^{*}(u)\right| \leq L_{3} \forall u$ $\in \Omega$. Thus, $\forall u \in \Omega$, we have

$$
\begin{aligned}
& \left|T_{u}(t)\right| \leq \int_{t_{k}}^{t} \frac{(t-s)^{\alpha-1}}{\Gamma(\alpha)}|f(s, u(s))+A(s) u(s)| d s \\
& +\left|\lambda_{1}(t)\right| \int_{t_{p}}^{T} \frac{(T-s)^{\alpha-1}}{\Gamma(\alpha)}|f(s, u(s))+A(s) u(s)| d s \\
& +\left|\lambda_{2}(t)\right| \int_{t_{p}}^{T} \frac{(T-s)^{\alpha-2}}{\Gamma(\alpha-1)}|f(s, u(s))+A(s) u(s)| d s \\
& +\sum_{i=1}^{k}\left[\int_{t_{i-1}}^{t_{i}} \frac{\left(t_{i}-s\right)^{\alpha-1}}{\Gamma(\alpha)}|f(s, u(s))+A(s) u(s)| d s+\left|I_{i}\left(u\left(t_{i}\right)\right)\right|\right] \\
& +\sum_{i=1}^{k-1}\left(t_{k}-t_{i}\right)\left[\int_{t_{i-1}}^{t_{i}} \frac{\left(t_{i}-s\right)^{\alpha-2}}{\Gamma(\alpha-1)}|f(s, u(s))+A(s) u(s)| d s+\left|I_{i}^{*}\left(u\left(t_{i}\right)\right)\right|\right] \\
& +\sum_{i=1}^{k}\left(t-t_{k}\right)\left[\int_{t_{i-1}}^{t_{i}} \frac{\left(t_{i}-s\right)^{\alpha-2}}{\Gamma(\alpha-1)}|f(s, u(s))+A(s) u(s)| d s+\left|I_{i}^{*}\left(u\left(t_{i}\right)\right)\right|\right] \\
& +\left|\lambda_{1}(t)\right| \sum_{i=1}^{p}\left[\int_{t_{i-1}}^{t_{i}} \frac{\left(t_{i}-s\right)^{\alpha-1}}{\Gamma(\alpha)}|f(s, u(s))+A(s) u(s)| d s+\left|I_{i}\left(u\left(t_{i}\right)\right)\right|\right] \\
& +\left|\lambda_{1}(t)\right| \sum_{i=1}^{p-1}\left(t_{p}-t_{i}\right)\left[\int_{t_{i-1}}^{t_{i}} \frac{\left(t_{i}-s\right)^{\alpha-2}}{\Gamma(\alpha-1)}|f(s, u(s))+A(s) u(s)| d s+\left|I_{i}^{*}\left(u\left(t_{i}\right)\right)\right|\right] \\
& +\sum_{i=1}^{p}\left[\left|\lambda_{3}(t)\right|+\left|\lambda_{1}(t)\right| t_{p}\right]\left[\int_{t_{i-1}}^{t_{i}} \frac{\left(t_{i}-s\right)^{\alpha-2}}{\Gamma(\alpha-1)}|f(s, u(s))+A(s) u(s)| d s+\left|I_{i}^{*}\left(u\left(t_{i}\right)\right)\right|\right] \\
& \leq\left(L_{1}+A_{1} r\right) \int_{t_{k}}^{t} \frac{(t-s)^{\alpha-1}}{\Gamma(\alpha)} d s+\left|\lambda_{1}(t)\right|\left(L_{1}+A_{1} r\right) \int_{t_{p}}^{T} \frac{(T-s)^{\alpha-1}}{\Gamma(\alpha)} d s \\
& +\left|\lambda_{2}(t)\right|\left(L_{1}+A_{1} r\right) \int_{t_{p}}^{T} \frac{(T-s)^{\alpha-2}}{\Gamma(\alpha-1)} d s+\sum_{i=1}^{p}\left[\left(L_{1}+A_{1} r\right) \int_{t_{i-1}}^{t_{i}} \frac{\left(t_{i}-s\right)^{\alpha-1}}{\Gamma(\alpha)} d s+L_{2}\right] \\
& +\sum_{i=1}^{p-1} T\left[\left(L_{1}+A_{1} r \int_{t_{i-1}}^{t_{i}} \frac{\left(t_{i}-s\right)^{\alpha-2}}{\Gamma(\alpha-1)} d s+L_{3}\right]+\sum_{i=1}^{p} T\left[\left(L_{1}+A_{1} r\right) \int_{t_{i-1}}^{t_{i}} \frac{\left(t_{i}-s\right)^{\alpha-2}}{\Gamma(\alpha-1)} d s+L_{3}\right]\right. \\
& +\left|\lambda_{1}(t)\right| \sum_{i=1}^{p}\left[\left(L_{1}+A_{1} r\right) \int_{t_{i-1}}^{t_{i}} \frac{\left(t_{i}-s\right)^{\alpha-1}}{\Gamma(\alpha)} d s+L_{2}\right] \\
& +\left|\lambda_{1}(t)\right| \sum_{i=1}^{p-1} T\left[\left(L_{1}+A_{1} r\right) \int_{t_{i-1}}^{t_{i}} \frac{\left(t_{i}-s\right)^{\alpha-2}}{\Gamma(\alpha-1)} d s+L_{3}\right] \\
& +\sum_{i=1}^{p}\left[\left|\lambda_{3}(t)\right|+T\left|\lambda_{1}(t)\right|\right] T\left[\left(L_{1}+7 A_{1} r\right) \int_{t_{i-1}}^{t_{i}} \frac{\left(t_{i}-s\right)^{\alpha-2}}{\Gamma(\alpha-1)} d s+L_{3}\right] \\
& \leq \frac{\left[(2 p-1)\left(1+T\left|\lambda_{1}(t)\right|\right)+p\left|\lambda_{3}(t)\right|+\left|\lambda_{2}(t)\right|\right] T^{\alpha-1}\left(L_{1}+A_{1} r\right)}{\Gamma(\alpha)}+\left(1+\left|\lambda_{1}(t)\right|\right) p L_{2} \\
& +\frac{(1+p)\left(1+\left|\lambda_{1}(t)\right| T^{\alpha}\left(L_{1}+A_{1} r\right)\right.}{\Gamma(\alpha+1)}+\left[(2 p-1)\left(1+T\left|\lambda_{1}(t)\right|\right)+p\left|\lambda_{3}(t)\right|\right] L_{3} .
\end{aligned}
$$

Since $t \in[0, T]$, therefore there exists a positive constant $L$, such that $\|T u\| \leq L$, which implies that the operator $T$ is uniformly bounded. 
On the other hand, for any $t \in J_{k}, 0 \leq k \leq p$, we have

$$
\begin{aligned}
& \left|\left(T_{u}\right)^{\prime}(t)\right| \leq \int_{t_{k}}^{t} \frac{(t-s)^{\alpha-2}}{\Gamma(\alpha-1)}|f(s, u(s))+A(s) u(s)| d s \\
& +\lambda_{4} \int_{t_{p}}^{T} \frac{(T-s)^{\alpha-1}}{\Gamma(\alpha)}|f(s, u(s))+A(s) u(s)| d s \\
& +\lambda_{5} \int_{t_{p}}^{T} \frac{(T-s)^{\alpha-2}}{\Gamma(\alpha-1)}|f(s, u(s))+A(s) u(s)| d s \\
& +\sum_{i=1}^{p}\left[\int_{t_{i-1}}^{t_{i}} \frac{\left(t_{i}-s\right)^{\alpha-2}}{\Gamma(\alpha-1)}|f(s, u(s))+A(s) u(s)| d s+\left|I_{i}^{*}\left(u\left(t_{i}\right)\right)\right|\right] \\
& +\lambda_{4} \sum_{i=1}^{p}\left[\int_{t_{i-1}}^{t_{i}} \frac{\left(t_{i}-s\right)^{\alpha-1}}{\Gamma(\alpha)}|f(s, u(s))+A(s) u(s)| d s+\left|I_{i}\left(u\left(t_{i}\right)\right)\right|\right] \\
& +\lambda_{4} \sum_{i=1}^{p-1}\left(t_{p}-t_{i}\right)\left[\int_{t_{i-1}}^{t_{i}} \frac{\left(t_{i}-s\right)^{\alpha-2}}{\Gamma(\alpha-1)}|f(s, u(s))+A(s) u(s)| d s+\left|I_{i}^{*}\left(u\left(t_{i}\right)\right)\right|\right] \\
& +\sum_{i=1}^{p}\left|\frac{(a+b) T+(b c-a d)\left(T-t_{p}\right)}{T \Lambda}\right| \\
& \times\left[\int_{t_{i-1}}^{t_{i}} \frac{\left(t_{i}-s\right)^{\alpha-2}}{\Gamma(\alpha-1)}|f(s, u(s))+A(s) u(s)| d s+\left|I_{i}^{*}\left(u\left(t_{i}\right)\right)\right|\right] \\
& \leq\left(L_{1}+A_{1} r\right) \int_{t_{k}}^{t} \frac{(t-s)^{\alpha-2}}{\Gamma(\alpha-1)} d s+\lambda_{4}\left(L_{1}+A_{1} r\right) \int_{t_{p}}^{T} \frac{(T-s)^{\alpha-1}}{\Gamma(\alpha)} d s \\
& +\lambda_{5}\left(L_{1}+A_{1} r\right) \int_{t_{p}}^{T} \frac{(T-s)^{\alpha-2}}{\Gamma(\alpha-1)} d s+\sum_{i=1}^{p}\left[\left(L_{1}+A_{1} r\right) \int_{t_{i-1}}^{t_{i}} \frac{\left(t_{i}-s\right)^{\alpha-2}}{\Gamma(\alpha-1)} d s+L_{3}\right] \\
& +\lambda_{4} \sum_{i=1}^{p}\left[\left(L_{1}+A_{1} r\right) \int_{t_{i-1}}^{t_{i}} \frac{\left(t_{i}-s\right)^{\alpha-1}}{\Gamma(\alpha)} d s+L_{2}\right]+\lambda_{4} \sum_{i=1}^{p-1}\left[\left(L_{1}+A_{1} r\right) \int_{t_{i-1}}^{t_{i}} \frac{\left(t_{i}-s\right)^{\alpha-2}}{\Gamma(\alpha-1)} d s+L_{3}\right] \\
& +\sum_{i=1}^{p}\left(\lambda_{5}+T \lambda_{4}\right)\left[\left(L_{1}+A_{1} r\right) \int_{t_{i-1}}^{t_{i}} \frac{\left(t_{i}-s\right)^{\alpha-2}}{\Gamma(\alpha-1)} d s+L_{3}\right] \\
& \leq \frac{(1+p) \lambda_{4} T^{\alpha}\left(L_{1}+A_{1} r\right)}{\Gamma(\alpha+1)}+\left[(1+p)\left(1+\lambda_{5}\right)+(1+p T) \lambda_{4}\right] \frac{T^{\alpha-1}\left(L_{1}+A_{1} r\right)}{\Gamma(\alpha)} \\
& +p \lambda_{4} L_{2}+\left[p+(1+p T) \lambda_{4}+(1+p) \lambda_{5}\right] L_{3}:=\bar{L} .
\end{aligned}
$$

Hence, for $t_{1}, t_{2} \in J_{k}, t_{1}<t_{2}, 0 \leq k \leq p$, we have

$$
\left|(T u)\left(t_{2}\right)-(T u)\left(t_{1}\right)\right| \leq \int_{t_{1}}^{t_{2}}\left|(T u)^{\prime}(s)\right| d s \leq \bar{L}\left(t_{2}-t_{1}\right)
$$

which implies that $T$ is equicontinuous on all $J_{k}, k=0,1,2, \ldots, p$. Thus, by the Arzela-Ascoli Theorem, the operator $T: P C(J, \mathbb{R}) \rightarrow P C(J, \mathbb{R})$ is completely continuous.

We need the following known results to prove the existence of solutions for (1.1).

Theorem 2.1 [40]Let E be a Banach space. Assume that $\Omega$ is an open bounded subset of $E$ with $\theta \in \Omega$ and let $T: \bar{\Omega} \rightarrow$ E be a completely continuous operator such that

$$
\|T u\| \leq\|u\|, \quad \forall u \in \partial \Omega .
$$

Then $T$ has a fixed point in $\bar{\Omega}$.

Theorem 2.2 Let $\lim _{u \rightarrow 0} \frac{f(t, u)}{u}=0, \lim _{u \rightarrow 0} \frac{I_{k}(u)}{u}=0$ and $\lim _{u \rightarrow 0} \frac{I_{k}^{*}(u)}{u}=0$, then the problem (1.1) has at least one solution.

Proof. In view of $\lim _{u \rightarrow 0} \frac{f(t, u)}{u}=0, \lim _{u \rightarrow 0} \frac{I_{k}(u)}{u}=0$ and $\lim _{u \rightarrow 0} \frac{I_{k}^{*}(u)}{u}=0$, then there exists a constant $r>0$ such that $|f(t, u)| \leq \delta_{1}|u|,\left|I_{k}(u)\right| \leq \delta_{2}|u|$ and $\left|I_{k}^{*}(u)\right| \leq \delta_{3}|u|$ for $0<1$ $u \mid<r$, 
where $\delta_{i}>0(i=1,2,3)$ satisfy the inequality

$$
\begin{aligned}
& \sup _{t \in J}\left\{\frac{(1+p)\left(1+\left|\lambda_{1}(t)\right|\right) T^{\alpha}\left(\delta_{1}+A_{1}\right)}{\Gamma(\alpha+1)}+\frac{\left[(2 p-1)\left(1+T\left|\lambda_{1}(t)\right|\right)+p\left|\lambda_{3}(t)\right|+\left|\lambda_{2}(t)\right| \mid\right]^{\alpha-1}\left(\delta_{1}+A_{1}\right)}{\Gamma(\alpha)}\right. \\
& \left.+\left(1+\left|\lambda_{1}(t)\right|\right) p \delta_{2}+\left[(2 p-1)\left(1+T\left|\lambda_{1}(t)\right|\right)+p\left|\lambda_{3}(t)\right|\right] \delta_{3}\right\} \leq 1 .
\end{aligned}
$$

Let us set $\Omega=\{u \in \operatorname{PC}(J, \mathbb{R}) \mid\|u\|<r\}$ and take $u \in \operatorname{PC}(J, \mathbb{R})$ such that $\|u\|=r$, that is, $u \in \partial \Omega$. Then, by the process used to obtain (2.2), we have

$$
\begin{aligned}
|T u(t)| & \leq \sup _{t \in J}\left\{\frac{(1+p)\left(1+\left|\lambda_{1}(t)\right| \mid T^{\alpha}\left(\delta_{1}+A_{1}\right)\right.}{\Gamma(\alpha+1)}+\frac{\left[(2 p-1)\left(1+T\left|\lambda_{1}(t)\right|\right)+p\left|\lambda_{3}(t)\right|+\left|\lambda_{2}(t)\right| \mid T^{\alpha-1}\left(\delta_{1}+A_{1}\right)\right.}{\Gamma(\alpha)}\right. \\
& +\left(1+\left|\lambda_{1}(t)\right|\right) \phi \delta_{2}+\left[(2 p-1)\left(1+T\left|\lambda_{1}(t)\right|\right)+p\left|\lambda_{3}(t)\right| \mid \delta_{3}\right\}\|u\| .
\end{aligned}
$$

Thus, it follows that $\|T u\| \leq\|u\|, u \in \partial \Omega$. Therefore, by Theorem 2.1, the operator $T$ has at least one fixed point, which in turn implies that the problem (1.1) has at least one solution $u \in \bar{\Omega}$.

Theorem 2.3 Assume that there exist positive constants $K_{i}(i=1,2,3)$ such that

$$
|f(t, u)-f(t, v)| \leq K_{1}|u-v|, \quad\left|I_{k}(u)-I_{k}(v)\right| \leq K_{2}|u-v|, \quad\left|I_{k}^{*}(u)-I_{k}^{*}(v)\right| \leq K_{3}|u-v|,
$$

for $t \in J, u, v \in \mathbb{R}$ and $k=1,2, \ldots, p$.

Then the problem (1.1) has a unique solution if $\mathcal{H}<1$, where

$$
\begin{aligned}
\mathcal{H} & =\max _{t \in J}\left\{\frac{(1+p)\left(1+\left|\lambda_{1}(t)\right|\right) T^{\alpha}\left(K_{1}+A_{1}\right)}{\Gamma(\alpha+1)}+\left[(2 p-1)\left(1+T\left|\lambda_{1}(t)\right|\right)+p\left|\lambda_{3}(t)\right|\right] K_{3}\right. \\
& \left.+\left(1+\left|\lambda_{1}(t)\right|\right) p K_{2}+\frac{\left[(2 p-1)\left(1+T\left|\lambda_{1}(t)\right|\right)+p\left|\lambda_{3}(t)\right|+\left|\lambda_{2}(t)\right|\right] T^{\alpha-1}\left(K_{1}+A_{1}\right)}{\Gamma(\alpha)}\right\} .
\end{aligned}
$$

Proof. Denote $F(s)=|f(s, u(s))-f(s, v(s))|+|A(s) u(s)-A(s) v(s)|$.

For $u, v \in P C(J, \mathbb{R})$, we have

$$
\begin{aligned}
& |(T u)(t)-(T v)(t)| \\
\leq & \int_{t_{k}}^{t} \frac{(t-s)^{\alpha-1}}{\Gamma(\alpha)} F(s) d s+\left|\lambda_{1}(t)\right| \int_{t_{p}}^{T} \frac{(T-s)^{\alpha-1}}{\Gamma(\alpha)} F(s) d s+\left|\lambda_{2}(t)\right| \int_{t_{p}}^{T} \frac{(T-s)^{\alpha-2}}{\Gamma(\alpha-1)} F(s) d s \\
& +\sum_{i=1}^{k}\left[\int_{t_{i-1}}^{t_{i}} \frac{\left(t_{i}-s\right)^{\alpha-1}}{\Gamma(\alpha)} F(s) d s+\left|I_{i}\left(u\left(t_{i}\right)\right)-I_{i}\left(v\left(t_{i}\right)\right)\right|\right]+\sum_{i=1}^{k-1}\left(t_{k}-t_{i}\right)\left[\int_{t_{i-1}}^{t_{i}} \frac{\left(t_{i}-s\right)^{\alpha-2}}{\Gamma(\alpha-1)} F(s) d s\right. \\
& \left.+\left|I_{i}^{*}\left(u\left(t_{i}\right)\right)-I_{i}^{*}\left(v\left(t_{i}\right)\right)\right|\right]+\sum_{i=1}^{k}\left(t-t_{k}\right)\left[\int_{t_{i-1}}^{t_{i}} \frac{\left(t_{i}-s\right)^{\alpha-2}}{\Gamma(\alpha-1)} F(s) d s+\left|I_{i}^{*}\left(u\left(t_{i}\right)\right)-I_{i}^{*}\left(v\left(t_{i}\right)\right)\right|\right] \\
& +\left|\lambda_{1}(t)\right| \sum_{i=1}^{p}\left[\int_{t_{i-1}}^{t_{i}} \frac{\left(t_{i}-s\right)^{\alpha-1}}{\Gamma(\alpha)} F(s) d s+\left|I_{i}\left(u\left(t_{i}\right)\right)-I_{i}\left(v\left(t_{i}\right)\right)\right|\right] \\
& +\left|\lambda_{1}(t)\right| \sum_{i=1}^{p-1}\left(t_{p}-t_{i}\right)\left[\int_{t_{i-1}}^{t_{i}} \frac{\left(t_{i}-s\right)^{\alpha-2}}{\Gamma(\alpha-1)} F(s) d s+\left|I_{i}^{*}\left(u\left(t_{i}\right)\right)-I_{i}^{*}\left(v\left(t_{i}\right)\right)\right|\right] \\
& +\sum_{i=1}^{p}\left[\left|\lambda_{3}(t)\right|+\left|\lambda_{1}(t)\right| t_{p}\right]\left[\int_{t_{i-1}}^{t_{i}} \frac{\left(t_{i}-s\right)^{\alpha-2}}{\Gamma(\alpha-1)} F(s) d s+\left|I_{i}^{*}\left(u\left(t_{i}\right)\right)-I_{i}^{*}\left(v\left(t_{i}\right)\right)\right|\right] \\
\leq & \left\{\frac{(1+p)\left(1+\left|\lambda_{1}(t)\right|\right) T^{\alpha}\left(K_{1}+A_{1}\right)}{\Gamma(\alpha+1)}+\frac{\left[(2 p-1)\left(1+T\left|\lambda_{1}(t)\right|\right)+p\left|\lambda_{3}(t)\right|+\left|\lambda_{2}(t)\right|\right] T^{\alpha-1}\left(K_{1}+A_{1}\right)}{\Gamma(\alpha)}\right. \\
& \left.+\left(1+\left|\lambda_{1}(t)\right|\right) p K_{2}+\left[(2 p-1)\left(1+T\left|\lambda_{1}(t)\right|\right)+p\left|\lambda_{3}(t)\right|\right] K_{3}\right\}\|u-v\| .
\end{aligned}
$$

Thus, we obtain $\|T u-T v\| \leq \mathcal{H}\|u-v\|$, where $\mathcal{H}$ is given by (2.5). As $\mathcal{H}<1$, therefore, $T$ is a contraction. Thus, the conclusion of the theorem follows by the contraction mapping principle. This completes the proof. 


\section{Examples}

Example 3.1 Consider the following fractional order impulsive mixed boundary value problem

$$
\left\{\begin{array}{l}
{ }^{C} D^{\alpha} u(t)=\frac{1}{3} \cos t+e^{u^{3}(t)}-1, \quad 0<t<T, \quad t \neq t_{1}, \quad 0<t_{1}<T \\
\Delta u\left(t_{1}\right)=2 \ln \left(1+u^{2}\left(t_{1}\right)\right), \quad \Delta u^{\prime}\left(t_{1}\right)=\left[1-\cos u\left(t_{1}\right)\right]^{2} \\
T u^{\prime}(0)=-\frac{1}{2} u(0)-\frac{1}{3} u(T), \quad T u^{\prime}(T)=\frac{1}{4} u(0)+\frac{1}{5} u(T)
\end{array}\right.
$$

where

$1<\alpha \leq 2, A(t)=\frac{1}{3} \cos t, f(t, u)=e^{u^{3}}-1, I_{1}(u)=2 \ln \left(1+u^{2}\right), I_{1}^{*}(u)=(1-\cos u)^{2}, a=\frac{1}{2}, b=\frac{1}{3}, c=\frac{1}{4}, d=\frac{1}{5}$ and $p=1$.

Clearly all the assumptions of Theorem 2.2 hold. Thus, the conclusion of Theorem 2.2 applies and the impulsive fractional mixed boundary value problem (3.1) has at least one solution.

\section{Author details}

${ }^{1}$ School of Mathematics and Computer Science, Shanxi Normal University, Linfen, Shanxi 041004, P. R. China ${ }^{2}$ Department of Mathematics, China University of Petroleum, Qingdao, Shandong 266555, P. R. China

\section{Authors' contributions}

GW and LZ completed the main part of this paper, GS corrected the main theorems and gave an example. All authors read and approved the final manuscript.

\section{Competing interests}

The authors declare that they have no competing interests.

Received: 15 November 2011 Accepted: 14 February 2012 Published: 14 February 2012

\section{References}

1. Sabatier, J, Agrawal, OP, Machado, JAT (eds.): Advances in Fractional Calculus: Theoretical Developments and Applications in Physics and Engineering. Springer, Dordrecht (2007)

2. Podlubny, I: Fractional Differential Equations, Mathematics in Science and Engineering. Academic Press, New York (1999)

3. Kilbas, AA, Srivastava, HM, Trujillo, Jj: Theory and Applications of Fractional Differential Equations. In North-Holland Mathematics Studies, vol. 204,Elsevier, Amsterdam (2006)

4. Samko, SG, Kilbas, AA, Marichev, OI: Fractional Integrals and Derivatives. Theory and Applications. Gordon and Breach, Yverdon (1993)

5. Lakshmikantham, V, Leela, S, Vasundhara Devi, J: Theory of Fractional Dynamic Systems. Cambridge Academic Publishers, Cambridge (2009)

6. He, J: Approximate analytical solution for seepage flow with fractional derivatives in porous media. Comput Methods Appl Mech Eng. 167, 57-68 (1998). doi:10.1016/S0045-7825(98)00108-X

7. El-Sayed, AMA: Fractional order diffusion wave equation. Int J Theor Phys. 35, 311-322 (1996). doi:10.1007/BF02083817

8. Gafiychuk, V, Datsun, B, Meleshko, V: Mathematical modeling of time fractional reaction-diffusion systems. J Comput Appl Math. 220, 215-225 (2008). doi:10.1016/j.cam.2007.08.011

9. Metzler, R, Klafter, J: The restaurant at the end of the random walk: recent developments in the description of anomalous transport by fractional dynamics. J Phys Math Gen. 37, R161-208 (2004). doi:10.1088/0305-4470/37/31/R01

10. Delbosco, D, Rodino, L: Existence and uniqueness for a nonlinear fractional differential equation. J Math Anal Appl. 204, 609-625 (1996). doi:10.1006/jmaa.1996.0456

11. He, J: Some applications of nonlinear fractional differential equations and their approximations. Bull Sci Technol. 15, 86-90 (1999)

12. Metzler, R, Schick, W, Kilian, H-G, Nonnenmacher, TF: Relaxation in filled polymers: a fractional calculus approach. J Chem Phys. 103, 7180-7186 (1995). doi:10.1063/1.470346

13. Ryabov, YaE, Puzenko, A: Damped oscillations in view of the fractional oscillator equation. Phys Rev B. 66,184201 (2002)

14. Agarwal, RP, Zhou, Y, He, Y: Existence of fractional neutral functional differential equations. Comput Math Appl. 59, 1095-1100 (2010). doi:10.1016/j.camwa.2009.05.010

15. Zhou, Y, Jiao, F: Nonlocal Cauchy problem for fractional evolution equations. Nonlinear Anal RWA. 11, 4465-4475 (2010). doi:10.1016/j.nonrwa.2010.05.029

16. Wang, J, Zhou, Y: A class of fractional evolution equations and optimal controls. Nonlinear Anal RWA. 12, 262-272 (2011). doi:10.1016/j.nonrwa.2010.06.013

17. Ahmad, B, Nieto, Jj: Existence results for a coupled system of nonlinear fractional differential equations with three-point boundary conditions. Comput Math Appl. 58, 1838-1843 (2009). doi:10.1016/j.camwa.2009.07.091

18. Ahmad, B, Sivasundaram, S: On four-point nonlocal boundary value problems of nonlinear integro-differential equations of fractional order. Appl Math Comput. 217, 480-487 (2010). doi:10.1016/j.amc.2010.05.080 
19. Ahmad, B, Alsaedi, A: Existence and uniqueness of solutions for coupled systems of higher order nonlinear fractional differential equations. Fixed Point Theory Appl 17 (2010). 2010 (Article ID 364560)

20. Nieto, Jj: Maximum principles for fractional differential equations derived from Mittag-Leffler functions. Appl Math Lett. 23, 1248-1251 (2010). doi:10.1016/j.aml.2010.06.007

21. Zhang, SQ: Positive solutions to singular boundary value problem for nonlinear fractional differential equation. Comput Math Appl. 59, 1300-1309 (2010). doi:10.1016/j.camwa.2009.06.034

22. Ntouyas, SK, Wang, G, Zhang, L: Positive solutions of arbitrary order nonlinear fractional differential equations with advanced arguments. Opuscula Mathematica. 31, 433-442 (2011)

23. Wang, G, Ntouyas, SK, Zhang, L: Positive solutions of the three-point boundary value problem for fractional-order differential equations with an advanced argument. Adv Diff Equ 2 (2011). 2011

24. Wang, G, Zhang, L, Ntouyas, SK: Multiplicity of positive solutions for fractional-order three-point boundary value problems. Commun Appl Nonlinear Anal to appear

25. Wang, G, Ahmad, B, Zhang, L: New existence results for nonlinear impulsive integro-differential equations of fractional order with nonlocal boundary conditions. Nonlinear Studies, to appear

26. Wang, G, Ahmad, B, Zhang, L: Some existence results for impulsive nonlinear fractional differential equations with mixed boundary conditions. Comput Math Appl. 62, 1389-1397 (2011). doi:10.1016/j.camwa.2011.04.004

27. Wang, G, Ahmad, B, Zhang, L: Impulsive anti-periodic boundary value problem for nonlinear differential equations of fractional order. Nonlinear Anal. 74, 792-804 (2011). doi:10.1016/j.na.2010.09.030

28. Zhang, L, Wang, G: Existence of solutions for nonlinear fractional differential equations with impulses and anti-periodic boundary conditions. Electron J Qual Theory Diff Equ. 7, 1-11 (2011)

29. Ahmad, B, Wang, G: A study of an impulsive four-point nonlocal boundary value problem of nonlinear fractional differential equations. Comput Math Appl. 62, 1341-1349 (2011). doi:10.1016/..camwa.2011.04.033

30. Ahmad, B, Nieto, JJ: Existence of solutions for impulsive anti-periodic boundary value problems of fractional order. Taiwanese J Math. 15, 981-993 (2011)

31. Ahmad, B, Sivasundaram, S: Existence results for nonlinear impulsive hybrid boundary value problems involving fractional differential equations. Nonlinear Anal Hybrid Syst. 3, 251-258 (2009). doi:10.1016/j.nahs.2009.01.008

32. Ahmad, B, Sivasundaram, S: Existence of solutions for impulsive integral boundary value problems of fractional order. Nonlinear Anal Hybrid Syst. 4, 134-141 (2010). doi:10.1016/j.nahs.2009.09.002

33. Agarwal, RP, Ahmad, B: Existence of solutions for impulsive anti-periodic boundary value problems of fractional semilinear evolution equations. Dyn Contin Discret Impulse Syst Ser A Math Anal. 18, 535-544 (2011)

34. Mophou, GM: Existence and uniqueness of mild solutions to impulsive fractional differential equations. Nonlinear Anal. 72, 1604-1615 (2010). doi:10.1016/j.na.2009.08.046

35. Tian, Y, Bai, Z: Existence results for the three-point impulsive boundary value problem involving fractional differential equations. Comput Math Appl. 59, 2601-2609 (2010). doi:10.1016/j.camwa.2010.01.028

36. Zhang, $X$, Huang, $X, L i u, Z$ : The existence and uniqueness of mild solutions for impulsive fractional equations with nonlocal conditions and infinite delay. Nonlinear Anal Hybrid Syst. 4, 775-781 (2010). doi:10.1016/j.nahs.2010.05.007

37. Jahanshahi, M: Reduction of the Neumann, Poincare and Robin-Zaremba boundary value problems for Laplace equation to the Dirichlet boundary value problem. Appl Comput Math. 6, 51-57 (2007)

38. Van den Berg, M, Gilkey, P, Kirsten, K, Kozlov, VA: Heat content asymptotics for Riemannian manifolds with Zaremba boundary conditions. Potential Anal. 26, 225-254 (2007). doi:10.1007/s11118-005-9001-1

39. Avramidi, IG: Heat kernel asymptotics of Zaremba boundary value problem. Math Phys Anal Geom. 7, 9-46 (2004)

40. Sun, JX: Nonlinear Functional Analysis and its Application. Science Press, Beijing (2008)

doi:10.1186/1687-2770-2012-17

Cite this article as: Zhang et al: On mixed boundary value problem of impulsive semilinear evolution equations of fractional order. Boundary Value Problems 2012 2012:17.

\section{Submit your manuscript to a SpringerOpen ${ }^{\ominus}$ journal and benefit from:}

Convenient online submission

- Rigorous peer review

- Immediate publication on acceptance

- Open access: articles freely available online

- High visibility within the field

- Retaining the copyright to your article

Submit your next manuscript at $\gg$ springeropen.com 